\title{
Leaf Trimming and High Temperature Regulation of Phytohormones and Polyamines in Creeping Bentgrass Leaves
}

\author{
Sanalkumar Krishnan, Yingmei Ma, and Emily Merewitz ${ }^{1}$ \\ Department of Plant, Soil and Microbial Sciences, Michigan State University, East Lansing, MI 48824
}

\begin{abstract}
AdDitional INDEX words. leaf wounding, heat stress, turfgrass, polyamines
Abstract. Mowing frequencies are associated with differences in disease susceptibility of turfgrasses, but how hormones respond to mowing practices are not fully understood. Two independent growth chamber experiments were conducted to determine how leaf trimming and heat stress play a role in modulating endogenous hormones within creeping bentgrass (Agrostis stolonifera) leaf tissues. The study also aimed to evaluate whether there are hormone changes at 0,15 , and 30 minutes after leaf trimming (wounding). The effects of trimming and temperature on sod plugs of creeping bentgrass 'Penncross' and 'Penn-G2' were investigated under optimal conditions $\left(23 / 20^{\circ} \mathrm{C}\right.$ day/night) and heat stress $\left(30 / 25{ }^{\circ} \mathrm{C}\right.$ day/night). Plants were 1$)$ untrimmed and sampled by plucking at the leaf base, 2) untrimmed and sampled by cutting at 0,15 , and 30 minutes, or 3 ) trimmed once every 3 days. Salicylic acid (SA), jasmonic acid (JA), abscisic acid (ABA), and indole-3-acetic acid (IAA) were generally greater in untrimmed plants compared with plants trimmed every 3 days under high temperature conditions. Zeatin riboside (ZR) was lower in untrimmed plants compared with plants trimmed every 3 days. JA and gibberellic acid (GA) accumulated to greater levels in the plants after 15 and 30 minutes of sampling. Polyamines (PAs) exhibited a transient increase in putrescine (Put) due to wounding. The results demonstrate the importance of research practices that consider the timing of sampling turfgrass plants for hormone analysis, help elucidate why mowing practices may play a role in stress susceptibility, and may be applicable to various studies related to leaf wounding.
\end{abstract}

Turfgrasses receive a high number of cultural inputs on a frequent basis for adequate performance and functionality. One of the inputs required for all turfgrass areas is frequent mowing. The most highly managed turfgrass areas, such as golf course putting greens or fairways, can be mowed as often as three times per week, daily, twice daily, or other similar rates (Turgeon, 2011). The importance of mowing practices is not reflected in the recent literature available related to how mowing practices affect turfgrass biochemistry and physiology. With modern advances in equipment and techniques to isolate and detect molecular and physiological changes, a greater understanding of the response of turfgrass species to mowing is possible and is needed. Turfgrass scientists too often have to rely on comparing physiological or hormonal responses to other crop species that are culturally managed highly differently than turfgrass species. No other major crop species is consistently mown as often as a turfgrass stand. Mowing practices alter turfgrass growth and architecture, but how hormones associated with growth changes such as cytokinins, GAs, and auxins in response to mowing are not well documented. Hormone responses in turfgrass species is a critical issue in turfgrass management, due to the widespread use of plant growth regulators and other substances altering plant growth physiology. An understanding of hormone responses is also critical for the development of germplasm with tolerance to abiotic and biotic stresses. Therefore, whether mowing causes hormonal

Received for publication 16 Oct. 2015. Accepted for publication 12 Nov. 2015. We thank the Michigan Turfgrass Foundation, AgBioResearch of Michigan State University, and the U.S. Department of Agriculture for funding and support.

1Corresponding author. E-mail: merewitz@msu.edu. fluctuations within the plant and whether the frequency of mowing has an effect on hormone profiles are two questions that need to be answered.

Mowing height and frequencies have been investigated for effects on turfgrass traits such as turfgrass performance, disease severity, and other treatments such as tolerance to abiotic stresses. Two major diseases, anthracnose (Colletotrichum cereal) and dollar spot (Sclerotinia homeocarpa), have been primarily focused on for evaluation of mowing practices on disease incidence and progression. Inguagiato et al. (2009) found that the hemibiotrophic anthracnose, a parasitic organism in living tissue that also can live in dead tissue, was significantly affected by mowing frequencies and heights. Overall, they concluded that raising the mowing height very small amounts can reduce disease severity. Mowing twice per day reduced disease severity compared with once per day on a limited number of days. However, more frequent mowing ( $6 \mathrm{~d} / \mathrm{week})$ compared with $(2 \mathrm{~d} /$ week $)$ increased disease occurrence of dollar spot on creeping bentgrass (Putman and Kaminski, 2011). Other reports are consistent in that low mowing heights tend to increase disease severity of anthracnose (Backman et al., 2002; Uddin and Soika, 2003). All of these diseases are most problematic in summer months under high temperatures (Turgeon, 2011). The major cause for mowing height differences are still not well understood and are often attributed to reduced photosynthetic potential, reduced carbohydrate reserves, reduced rooting, and other effects caused by extremely low mowing heights (Beard, 2002). Mowing frequency differences have been attributed to mechanical factors such as dew removal to reduce the wet environment often desired by turfgrass pathogens (Delvalle et al., 2011). Perhaps hormone regulation may play an important role in modulating turfgrass responses to 
disease incidence under varying heights of cut or frequencies of cut under optimal or high temperature conditions.

Mechanical plant leaf wounding causes both a local and systemic response. The major responses are for wound healing, reallocation of energy resources, or to prevent further attack, such as further insect herbivory or fungal invasion. These responses all require significant modification of many phytohormones and energy to be performed. The preventative responses may only serve as a benefit to the plant if the plants are subsequently attacked by a pathogen (Sanchez-Serrano, 2010). Jasmonic acid is the major hormone involved in mechanical wound responses as it highly regulates woundinduced gene expression leading to induced systemic resistance (ISR) and plays a major role in tolerance to necrotrophic pathogens. ISR is a mechanism that primes plants for enhanced disease or stress tolerance following a select set of environmental exposures, such as exposure to certain microbes or chemicals (Fu and Dong, 2013). Thus, determining whether turfgrasses may moderate these responses due to frequent mowing or continuously express these biochemical pathways is needed. If moderation of JA-regulated wound responses occur to save energy, frequently mowed turfgrass plants may be more susceptible to necrotrophic pathogens. Many necrotrophic pathogens, pathogens that kill host tissues, of highly managed turfgrasses do plague the turfgrass industry, such as dollar spot. SA acts antagonistically to JA or as an inhibitor to wounding responses in plant leaves. SA controls the systemic acquired resistance (SAR) pathway in order for a plant response to pathogen attack. SAR involves a local signal in response to pathogen attack that is converted to systemic defense mechanisms, such as the accumulation of pathogenesis-related proteins (Doares et al., 1995). How SA may be regulated by mowing practices or turfgrass leaf wounding has not yet been investigated.

In addition to other hormone responses due to mowing, how PAs may be associated with wounding responses of plants, particularly of turfgrasses is not well understood. PA biosynthesis and regulation of PA content is closely associated with the phytohormone pathways of SA and JA. PAs are also known to have plant growth regulator function and are classified by some as plant hormones (Davies, 1995). PAs have been shown to play a role in the defense against pathogens (Rossi et al., 2015) and may be directly involved in wounding responses (Lulai et al., 2015; Perez-Amador et al., 2002). Therefore, elucidation of PA responses to mowing is an initial step that could improve our understanding of growth regulation, pathogen defense, and other attributes such as abiotic stress related to mowing practices or leaf wounding. Determining whether PAs may respond to mowing will allow for more accurate comparisons inter- and intraexperimentally and enhance our ability to interpret results within the study of PAs in grass species. These results may also be useful for forage grass species or various sources of wounding stress other than mowing. In addition, how the major hormones described in this paper respond to heat stress in turfgrass species is not well documented.

Therefore, the objectives of the study were to evaluate hormone profiles in response to different leaf trimming practices to simulate mowing frequency under optimal or high temperature conditions and to understand hormone regulation in the early response to leaf wounding. The results of the study are important to better understand and interpret hormonal responses for the promotion of turfgrass science research and management.

\section{Materials and Methods}

Plant material and Growth COnditions. Sod plugs of creeping bentgrass were harvested from the Hancock Turfgrass Research Center in East Lansing, MI. Plugs (10.2 cm diameter) of creeping bentgrass 'Penncross' were harvested in May 2014 for Expt. 1 and 'Penn-G2' in Nov. 2014 for Expt. 2. Different cultivars were used due to differences in availability at the time of sod collection and for determination of consistency of response among different cultivars. The sod pieces were cut free of roots and soil to just below the crown and thatch layer. Then they were potted in polyvinyl chloride (PVC) tubes (10.2 $\mathrm{cm}$ diameter and $40 \mathrm{~cm}$ in length) containing sterilized native soil (a sandy loam, Typic Hapludult soil). PVC tubes were fit with mesh at the bottom to contain soil but allow plants to adequately drain. All plants were allowed to establish $\approx 1$ month. All plants were trimmed as needed to maintain a leaf canopy of $\approx 1.3 \mathrm{~cm}$ during establishment. Plants were fertilized equally with $500 \mathrm{~mL}$ of half strength Hoagland's solution twice per week throughout the duration of both experiments (Hoagland and Arnon, 1950). Two separate growth chamber units (TPRP series; Biochambers, Winnipeg, MB, Canada) were used for Expts. 1 and 2. The conditions were set to maintain similar conditions for both experiments with a relative humidity average of $65 \%$ and $700 \mu \mathrm{mol} \cdot \mathrm{m}^{-2} \cdot \mathrm{s}^{-1}$ light intensity for 14 h. Expt. 1 initiated on 19 June 2014 and ended on 11 Sept. 2014. Within this experiment, trimming treatments began on 19 June 2014 and were imposed until the conclusion of the study. Sampling under optimal temperatures $\left(23^{\circ} \mathrm{C}\right)$ began on 24 July 2014. Plants were then moved to high temperature conditions $\left(30{ }^{\circ} \mathrm{C}\right)$ on 7 Aug. 2014 and heat treatment was imposed until the end of the study on 11 Sept. 2014. Expt. 2 began on 15 Dec. 2014 and concluded on 19 Feb. 2015. Trimming treatments began on 5 Dec. 2014 and initial sampling on optimal temperature started on 22 Dec. 2014 and ended on 14 Jan. 2015. Plants were subjected to the same temperatures as in Expt. 1. Heat stress began on 22 Jan. 2015 and sampling began on 28 Jan. 2015 and continued until 19 Feb. 2015. A mild exposure to high temperatures was desired to not confound trimming response results to major differences in heat stress response between cultivars.

Trimming Treatments. Plants were 1) untrimmed and sampled by plucking at the leaf base; 2) trimmed $1 \times$ week and sampled by cutting at 0,15 , and 30 min after trimming; or 3 ) trimmed once every $3 \mathrm{~d}$. A total of six replications of each treatment were used in the study. In trim treatment 2 , the time point 0 min sampling is the same leaf tissue from the once per week trimming event meaning that the sampling was done at the same time as trimming. For treatment 3, plants were trimmed every $3 \mathrm{~d}$, but leaf tissue was only preserved once per week. Except for the untrimmed plants in treatment 1, the final canopy height of all plants after trimming and sampling treatments was $1.3 \mathrm{~cm}$. During trimming or time point sampling, leaf samples were saved for phytohormones and PA analysis. This was done by wrapping them in foil, placing them in liquid nitrogen, and preserving them at $-80{ }^{\circ} \mathrm{C}$ in a freezer.

Plants measured under optimal temperature $\left(23^{\circ} \mathrm{C}\right)$ were moved to high temperature conditions $\left(30^{\circ} \mathrm{C}\right)$ on the dates 
indicated above. Therefore, a total of 18 plants were used for Expts. 1 and 2, with six plants for each of the three trimming treatments. For all measurements requiring destructive sampling, untrimmed plants were sampled by plucking the second leaf on the stem that was fully expanded. Older leaves or immature leaves were not included in the analysis of these plants. All trimmed plants were sampled by cutting leaves evenly across the grass canopy with scissors. Plants used for the $0-, 15-$, and 30-min evaluation were trimmed once per week for sampling, meaning that the once per week trimming is the same plant tissue as the 0 -min treatment.

Physiological evaluation and hormone analysis. Turf quality (TQ), photochemical efficiency $\left(F_{\mathrm{v}} / F_{\mathrm{m}}\right)$, photochemical yield [quantum yield of regulated nonphotochemical energy loss in photosystem II (YII)] and sampling for hormone analysis were done on a weekly basis for Expt. 2. $F_{\mathrm{v}} / F_{\mathrm{m}}$ and YII were not available for Expt. 1 due to machine repair. For both experiments, TQ was rated on a 1 to 9 scale as described in Beard (2002), where $1=$ brown, desiccated turf and $9=$ healthy green turf. $F_{\mathrm{v}} / F_{\mathrm{m}}$ and YII were measured with a fluorimeter (OSp5; Opti-Sciences Inc., Hudson, $\mathrm{NH}$ ) on attached leaves.

Phytohormone extraction and quantification was based on the method used by Liu et al. (2012). About $200 \mathrm{mg}$ of frozen tissue samples was ground to a fine powder in liquid nitrogen using mortar and pestle. Extractions were handled in the same manner as described for kentucky bluegrass (Poa pratensis) in Krishnan and Merewitz (2015a) with modification to include GA isoforms. The internal standards for liquid chromatography (LC) analysis included $100 \mathrm{nmol}$ of deuterium-labeled ABA $\left({ }^{2} \mathrm{H}_{6}-\mathrm{ABA}\right)$ added at the time of extraction. Calibration standard solutions were prepared for SA, JA, ABA, IAAZR, GA, $\mathrm{GA}_{4}$, and $\mathrm{GA}_{20}$ and spiked with 100 nmol of ABA-d6. LC was carried out using an ultra high-performance LC-tandem mass spectrometry (Quattro Premier XE ACQUITY ${ }^{\circledR}$ Tandem Quadrupole; Waters, Milford, MA). It should be noted that ethylene is also an important hormone in wounding responses as it acts downstream of the JA pathway and PA pathways (Wang et al., 2002). However, due to the complexities of ethylene gas sampling we were not able to measure ethylene on clipped or plucked leaf samples in this study.

PA Content. In Expt. 1 optimal temperature samples were taken from plants sampled on 24 July 2014 and in Expt. 2 on 12 Dec. 2014. High temperature samples of PA content were from 21 Aug. 2014 in Expt. 1 and 2 Feb. 2015 in Expt. 2. Free PAs [Put, spermidine (Spd), and spermine (Spm)] of creeping bentgrass leaf samples for both experiments were extracted based on the method of Liu et al. (2011) and Oefner et al. (1992) with modification. Specifically, a fine slurry was obtained by adding $1 \mathrm{~mL}$ of $5 \%$ formic acid to the ground fine powder of $250 \mathrm{mg}$ fresh leaf tissue in liquid nitrogen. All ground sample slurry was collected in a 2-mL microcentrifuge tube and stored at $-80{ }^{\circ} \mathrm{C}$ until use. For further sample processing, the frozen samples were centrifuged at $4{ }^{\circ} \mathrm{C}$ with $20,817 g_{n}$ for $30 \mathrm{~min}$ after thawing at room temperature. The obtained supernatant containing PAs was adjusted for the $\mathrm{pH}$ to be higher than 12.0 with $2 \mathrm{M} \mathrm{NaOH}$. The samples were then dried in a vacuum concentrator (SpeedVac Savant SPD111V; Thermo Fisher Scientific, Waltham, MA) at room temperature.

To derivatize free PAs, the samples were resuspended in $500 \mu \mathrm{L}$ of $4 \%$ benzoyl chloride and incubated at $30^{\circ} \mathrm{C}$ for $2 \mathrm{~h}$ with shaking. After incubation, $500 \mu \mathrm{L}$ of saturated $\mathrm{NaCl}$ solution and $1 \mathrm{~mL}$ of dichloromethane (99\%) were added into the derivatized sample after centrifuging at $20,817 g_{n}$ for 20 min. After mixing well, the bottom layer containing derivatized PAs was collected and all of the salts were washed three times by pipetting. Finally, samples were lyophilized in a lyophilizer (Genesis Pilot Lyophilizer; SP Scientific, Warminster, PA) at room temperature. The lyophilized samples were resuspended in $60 \%$ methanol for high-performance LC coupled to electrospray ionization quadropole time-of-flight mass spectrometry analysis. The electrospray ionization was performed according to their mass charge ratio and mass separated ions were transmitted and detected by a photomultiplier detection system (Masslynx NTTM data system, Waters) by single ion recording techniques. The gradient mixture was separated under a multichannel analysis for data acquisition for total of 13 min running at filling speed of $10 \mu \mathrm{L} \cdot \mathrm{s}^{-1}$ under different retention time. External standards were prepared the same as samples described above. The reproducibility of standards under optimal conditions was verified by injection of mixtures of those three standards with $30 \mu \mathrm{M} \cdot \mathrm{L}^{-1}$ of each. A $60 \%$ methanol solvent was used as a blank between every six samples to show no carry over from previous samples solution.

Statistical ANALYSIS. Analysis of variance was employed to examine the effects of treatments on the response variables based on the general linear model procedure of SAS (version 9.1; SAS Institute, Cary, NC). Fisher's protected least significant difference (LSD) test at a $0.05 P$ level was used to detect differences between treatment means. LSD or letters are presented in the figures where statistical significance was detected.

\section{Results}

Physiological evaluation. TQ results of Expt. 1 were comparable to that of Expt. 2 in Winter 2015 (Fig. 1). In Expt. 1, TQ, $F_{\mathrm{v}} / F_{\mathrm{m}}$, and YII were only significantly different among treatments on 9 Sept. 2014 (data not shown). In Expt. 2, TQ did not significantly decline due to high temperatures for plants trimmed every $3 \mathrm{~d}$ and plants trimmed once weekly for sampling. TQ ratings were significantly lower for no trim treatments compared with the other treatments under high temperature conditions (Fig. 1A). In general, both yield and $F_{\mathrm{v}} / F_{\mathrm{m}}$ declined only slightly when exposed to higher temperatures compared with optimum temperature for all the treatments; thus only a very mild response to high temperatures was observed (Fig. 1B and C). Significant differences among trim treatments in YII were only detected during high temperatures on 28 Jan. 2015 (Fig. 1B). $F_{\mathrm{v}} / F_{\mathrm{m}}$ for untrimmed plants were significantly lower than plants trimmed every $3 \mathrm{~d}$, but were not significantly different from the plants trimmed only for weekly sampling on most days of high temperature conditions (Fig. 1C).

Hormone Content. The ranges in hormone contents detected were different between experiments and cultivars for all hormones measured. The SA levels increased from control optimal temperature levels under high temperature for some of the treatments, such as the untrimmed treatment and wounding time course treatments. This response was more pronounced in Expt. 2 for 'Penn-G2' compared with 'Penncross' in Expt. 1 (Fig. 2A and B). Under optimal temperature conditions, consistent significant differences between treatments for SA levels were not detected. Under high temperature conditions, SA levels of no trim plants were significantly higher than the other trim treatments on the same day for 'Penncross' in 

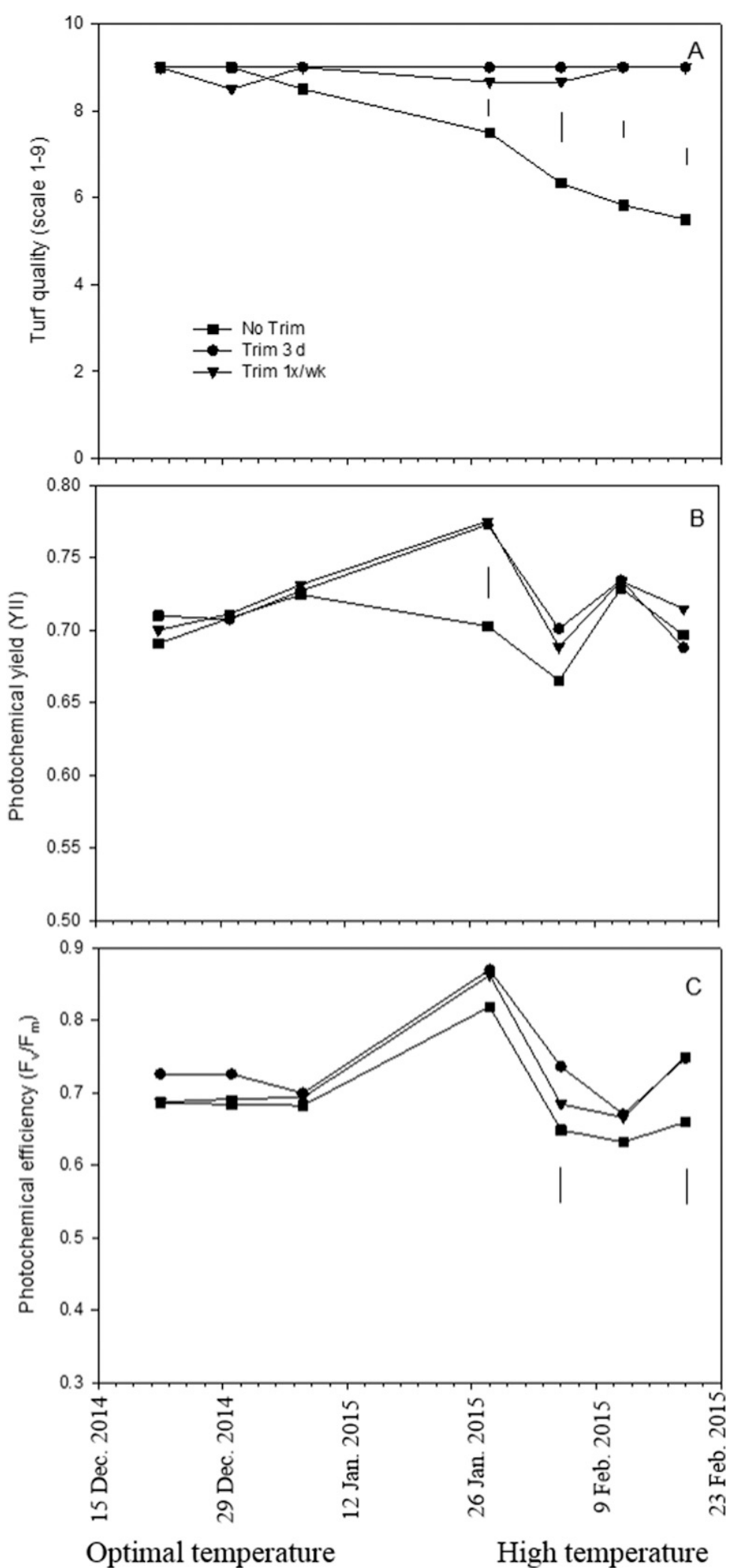

Fig. 1. (A) Turfgrass quality ( 1 to 9 scale with $1=$ dead plants and $9=$ healthy plants). (B) Photochemical yield (YII), and $(\mathbf{C})$ photochemical efficiency $\left(F_{\mathrm{v}} / F_{\mathrm{m}}\right)$ of creeping bentgrass plants exposed to trimming treatments combined with optimal or high temperature conditions of 'Penn-G2' plants in Expt. 2. Treatment means were separated using Fisher's protected least significant difference (LSD) $(\mathrm{n}=6)$ at $P \leq 0.05$. LSD values are only shown on days were significant differences were detected.
Expt. 1. Within Expt. 2 for 'Penn-G2', SA levels where significantly greater for the untrimmed treatment compared with the other treatments on all dates. Plants evaluated for time point-based changes in phytohormones at 0,15 , and $30 \mathrm{~min}$ did not exhibit significant change in SA content under either optimal or high temperature conditions.

JA content was higher for untrimmed plants compared with plants trimmed every $3 \mathrm{~d}$ on the last day evaluated at high temperature for both experiments (11 Sept. 2014 and 12 Feb. 2015). JA accumulation was found to be significantly higher for trims at 15 and 30 min compared with the other trim treatments under optimal and high temperature conditions for both of the experiments (Fig. 2C and D).

ABA levels were found to be a fresh weight of $\approx 50 \mathrm{ng} \cdot \mathrm{g}^{-1}$ for most of the treatments under optimal and high temperature conditions for both studies (Fig. 3A and B). The ABA levels did increase significantly more in untrimmed plants compared with the other trim treatments in response to higher temperature for both experiments. IAA accumulation patterns were similar to $\mathrm{ABA}$ in both studies (Fig. 3C and D). Under heat stress, plants accumulated higher levels of IAA after 15 or $30 \mathrm{~min}$; however, this was not significant for all days of sampling. Untrimmed plants had elevated IAA under heat stress compared with under optimal temperatures and higher IAA content compared with the other trim treatments on most days. Relative to the other hormones, ZR content was relatively low and did not exhibit large changes under optimal or high temperature conditions; however, ZR content was significantly lower due to heat stress for some of the trim treatments in both experiments (Fig. 3E and F). Within a given day of sampling, plants trimmed every 3 $\mathrm{d}$ showed higher levels of ZR compared with the other trim treatments in normal and heat stressed conditions on most days in both experiments.

$\mathrm{GA}_{1}$ levels increased due to heat stress for most treatments in both studies, with this response being more pronounced and consistent among treatments for 'Penn-G2' in Expt. 2 (Fig. 4A and B). $\mathrm{GA}_{1}$ generally decreased from 0 to $30 \mathrm{~min}$ after trimming on most days of sampling in both experiments. An increase in $\mathrm{GA}_{4}$ content was found due to trimming during the 0 - to 30 -min time point analysis for most days under optimal or heat stress conditions (Fig. 4C). However, this was not significant during heat stress of 'Penn-G2' in Expt. 2 (Fig. 4D). Heat stress caused an increase in $\mathrm{GA}_{20}$ content for most of the plant treatments compared with under 

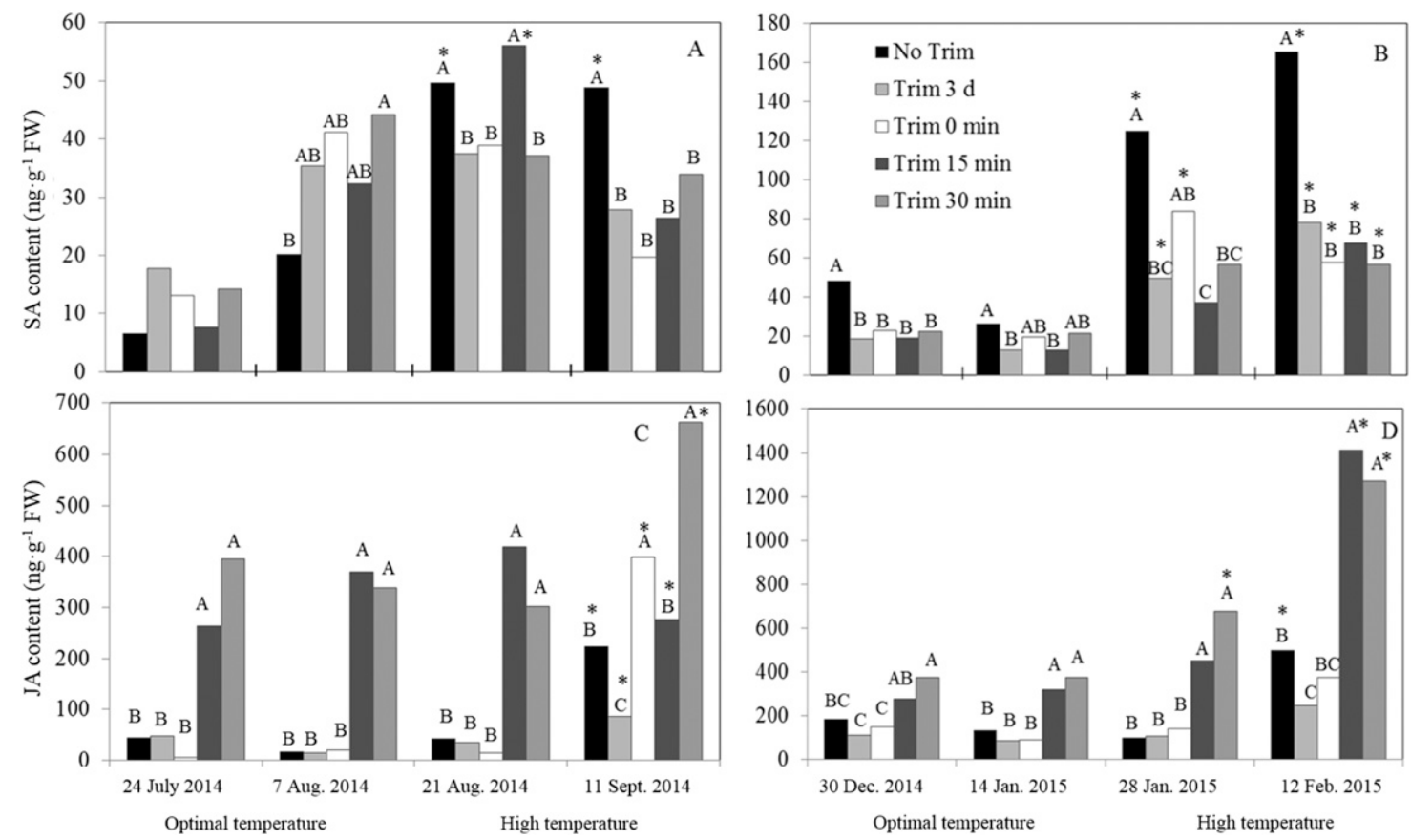

Fig. 2. Salicylic acid (SA) in (A) Expt. 1 'Penncross' and (B) Expt. 2 'Penn-G2'; and jasmonic acid (JA) content during (C) Expt. 1 'Penncross' and (D) Expt. 2 'Penn-G2' of creeping bentgrass plants exposed to trimming treatments combined with optimal or high temperature conditions. Different letters indicate significant differences among leaf trimming treatments within a given day. Asterisks indicate significant differences within a treatment comparing among temperature conditions. Letters and asterisks are based on separation of means using Fisher's protected least significant difference $(\mathrm{n}=6)$ at $P \leq 0.05$. Note scale differences on $y$ axis for all graphs used to visualize treatment differences.

optimal conditions; however, this was more pronounced for 'Penn-G2' in Expt. 2 (Fig. 4F). Within a sampling day, GA 20 accumulation was higher in plants trimmed every $3 \mathrm{~d}$ compared with no trim, trim 15, and trim 30 min under normal conditions in Expt. 1 (Fig. 4E).

PA CONTENT. Put content was significantly upregulated by heat stress for plants exposed to no trim, $0 \mathrm{~min}$, and $15 \mathrm{~min}$ in both Expts. 1 and 2, respectively (Fig. 5A and B). Within a given day and temperature treatment, a transient increase in Put was observed from 0,15 , to 30 min after trimming, with a significant increase in Put being observed at $15 \mathrm{~min}$. No significant difference was detected in Put content when comparing plants not trimmed to those that were trimmed every $3 \mathrm{~d}$. Spd content was lower in plants trimmed every $3 \mathrm{~d}$ compared with those left untrimmed. There was no consistent trend from 0 to 30 min after trimming between both experiments (Fig. 5C and D). No significant changes in Spm content were apparent either due to heat stress or due to trim treatment (Fig. 5E and F).

\section{Discussion}

Physiological parameters measured indicate there was mild indirect heat stress occurring in plants treated with high temperature compared with controls. Plants that were not trimmed appeared visually more damaged and had less photochemical health compared with trimmed plants. We suspect this could be because untrimmed plants have less leaf turnover into the crown/thatch area resulting in the older leaves remaining more visible on plant stems or due to changes in canopy microenvironments. Plants trimmed every $3 \mathrm{~d}$ had a more compact canopy which could affect canopy humidity levels and other factors. Thus, any modulation of hormone responses explicitly due to trimming treatments are likely most apparent under optimal conditions.

Hormone ranges detected when comparing Expts. 1 and 2 were significantly different. As hormones can have significant shifts in content due to many different environmental parameters, diurnal variation, seasonal variation, cultivar, and several other factors we do not expect that the relative levels of hormone between experiments is of concern (Alvim et al., 1976; Nováková et al., 2005). For Expt. 1 'Penncross' was used whereas in Expt. 2 'Penn-G2' was used. Similar variation has been observed for PA contents in other experiments (Papenfus et al., 2012). For the purposes of this study, what is most important is that the trends in hormone content changes due to temperature or to trimming were consistent between experiments and cultivars.

SA content of leaves remained relatively unchanged in response to wounding on most days when trimmed at 0,15 , and $30 \mathrm{~min}$. We found that JA increased dramatically $30 \mathrm{~min}$ after leaf wounding. Similar results were found in rice [Oryza sativa (Lee et al., 2004)]. In their study, a similar increase in JA within $1 \mathrm{~h}$ after wounding was observed and SA content decreased during this early response to wounding. The endogenous JA level was found to increase dramatically after mechanical wounding in a time dependent manner in sweetpotato [Ipomoea batatas (Rajendran et al., 2014)]. Thus, when evaluating hormones in turfgrass species, care needs to be taken regarding when tissue sampling is performed relative to trimming or mowing practices and that all plants are under the same trimming regime and timeframe. Hormone level fluctuations need to be considered when comparing hormone responses intra and interexperimentally. Immediate responses 


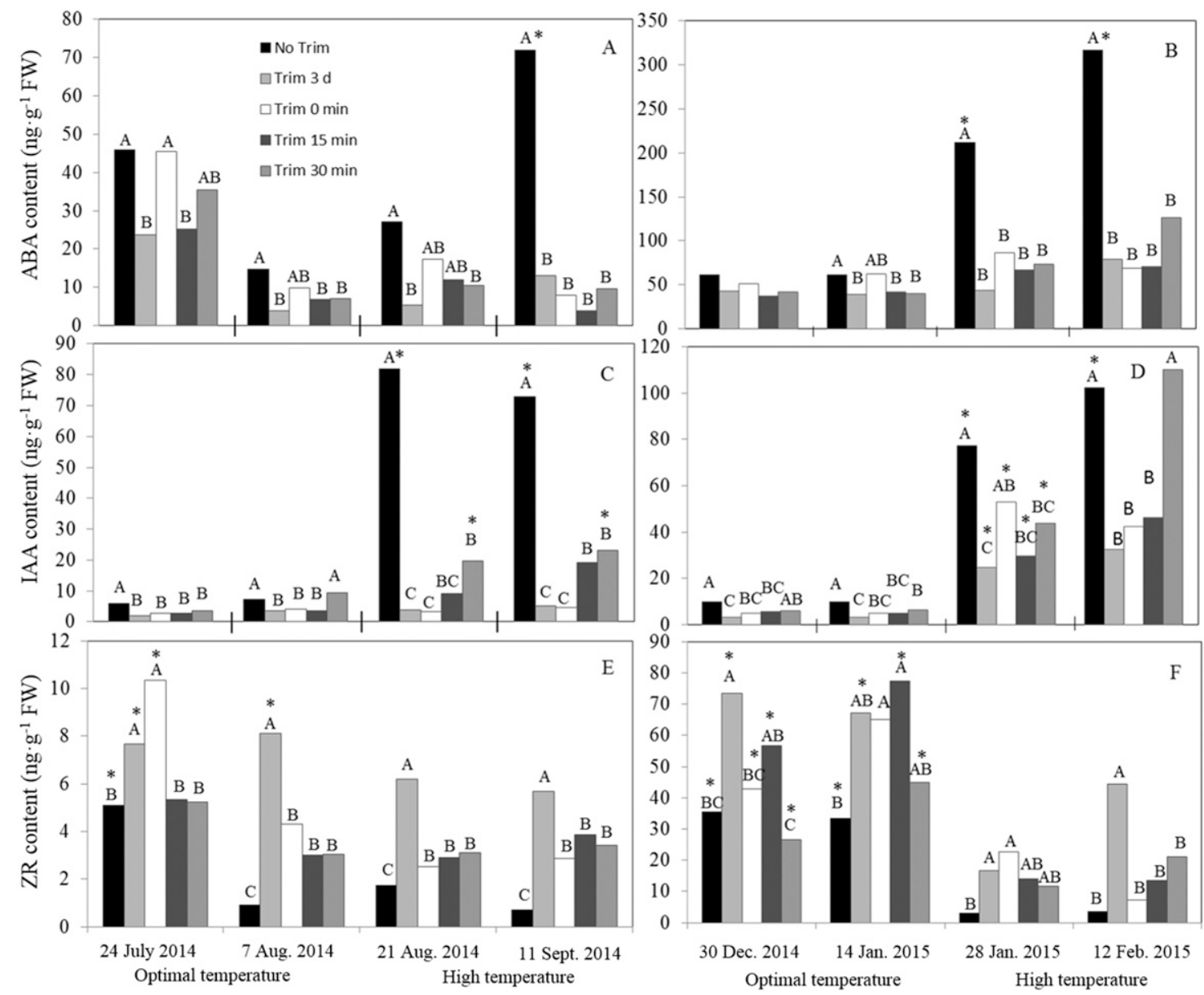

Fig. 3. Abscisic acid (ABA) in (A) Expt. 1 'Penncross' and (B) Expt. 2 'Penn-G2'; indole-3-acetic acid (IAA) content in (C) Expt. 1 'Penncross' and (D) Expt. 2 'Penn-G2'; and zeatin riboside (ZR) content in (E) Expt. 1 'Penncross' and (F) Expt. 2 'Penn-G2' of creeping bentgrass plants exposed to trimming treatments combined with optimal or high temperature conditions. Different letters indicate significant differences among leaf trimming treatments within a given day. Asterisks indicate significant differences within a treatment comparing among temperature conditions. Letters and asterisks are based on separation of means using Fisher's protected least significant difference $(\mathrm{n}=6)$ at $P \leq 0.05$. Note scale differences on $y$ axis for all graphs used to visualize treatment differences.

and signaling pathways of turfgrasses to leaf wounding should be investigated further.

In regard to trimming related to mowing frequency, creeping bentgrass leaves under high temperature conditions that were not trimmed had significantly greater SA content on most days compared with those that were trimmed every $3 \mathrm{~d}$ or once per week ( 0 min) under optimal conditions in Expt. 2 and under stress conditions in both experiments. A similar response occurred for JA but only on the last days of high temperature treatment for both experiments. A moderation of content of SA and JA by either frequent trimming could have potential impact on abiotic and biotic disease responses since these hormones control SAR and ISR pathways, respectively (Ton et al., 2002). Exogenous application of SA has been implicated in heat tolerance in creeping bentgrass (Larkindale and Huang, 2004) and in kentucky bluegrass [P. pratensis (He et al., 2005)]. Beyond exogenous application, little is known about SA responses in turfgrass species. In our previous studies, endogenous SA content was upregulated by drought and salt stresses (Krishnan and Merewitz, 2015a, 2015b). The results of this study suggest heat stress also may upregulate SA content in creeping bentgrass. The responses of SA and JA to trimming frequency and heat stress found here may play a role in why turfgrasses may be more or less susceptible to different diseases at different mowing heights and different frequencies. However, further evaluation and future work is needed to evaluate this observation more thoroughly.

$\mathrm{ABA}$ levels were not altered due to wounding responses during the 0 -to 30 -min time course sampling. ABA levels were generally higher in untrimmed plants compared with plants trimmed every $3 \mathrm{~d}$. As discussed above, this could be due to differences in heat stress experience. ABA is known to be a signaling compound for heat stress responses and can accumulate during heat stress (Kotak et al., 2007). However, on some days higher ABA was detected in untrimmed plants compared with plants trimmed every $3 \mathrm{~d}$ even under optimal conditions. Thus, ABA accumulation could be affected by trimming frequency. For clarity, a more detailed evaluation of ABA responses to trimming or mowing practices may be warranted.

The auxin, IAA, was greater in plants left untrimmed compared with most other trimming treatments on all sampling days. The reverse of this was true for the content of the cytokinin, ZR, since ZR was generally higher in plants trimmed every $3 \mathrm{~d}$ compared with plants left untrimmed. No consistent 

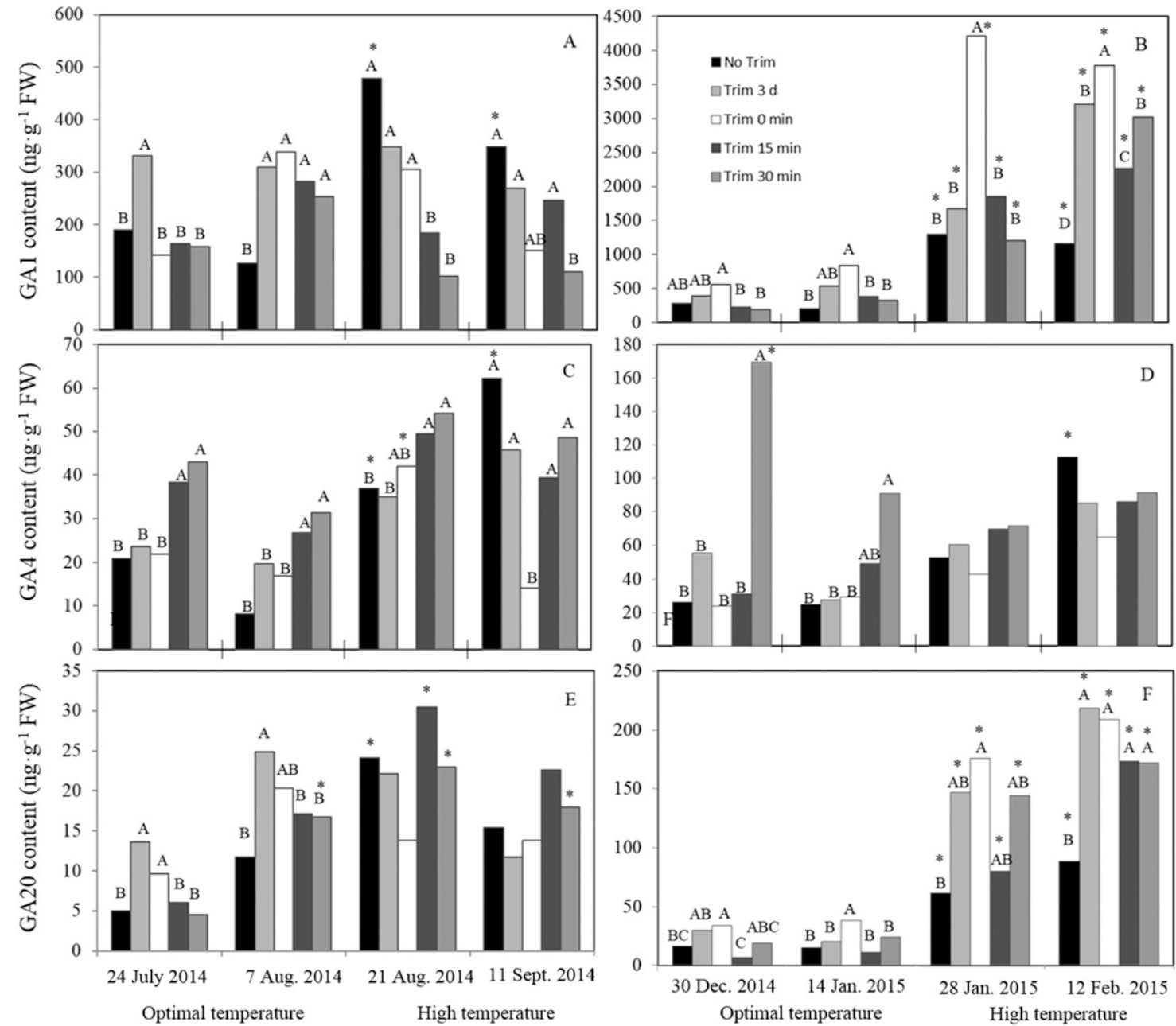

Fig. 4. Giberellic acid (GA) isoforms GA 1 in (A) Expt. 1 'Penncross' and (B) Expt. 2 'Penn-G2'; GA 4 in (C) Expt. 1 'Penncross' and (D) Expt. 2 'Penn-G2'; and $\mathrm{GA}_{20}$ in (E) Expt. 1 'Penncross' and (F) Expt. 2 'Penn-G2' of creeping bentgrass plants exposed to trimming treatments combined with optimal or high temperature conditions. Different letters indicate significant differences among leaf trimming treatments within a given day. Asterisks indicate significant differences within a treatment comparing among temperature conditions. Letters and asterisks are based on separation of means using Fisher's protected least significant difference $(\mathrm{n}=6)$ at $P \leq 0.05$. Note scale differences on $y$ axis for all graphs used to visualize treatment differences.

response was observed for ZR or IAA during the time course response to wounding, but on some days, IAA accumulation was stimulated after 15 or $30 \mathrm{~min}$ of wounding. These results are seemingly consistent with the known functions of auxins and cytokinins. Cytokinins function to stimulate cell division and interaction of cyokinins with auxins results in cellular differentiation (Davies, 1995). Leaf cutting may result in a higher demand for cytokinins to stimulate cell division in order for wound repair and regeneration. Untrimmed leaves likely do not require much additional cell division. Higher IAA content in plants left untrimmed could be related to lack of removal of IAA by trimming or due to leaf maturity. Young leaf tissues serve as a source of IAA to be transported throughout the plant (Petrásek and Friml, 2009). Regarding the time course sampling results, ZR is not well known to be among the earliest signals involved in wounding responses, but in some studies cytokinins do accumulate after wounding of various plant tissues (Crane and Ross, 1986; León et al., 2001). Auxins are reported to have an inhibitory effect on gene expression related to the wounding response. In some species IAA levels have decreased due to wounding and a promotion of IAA levels or return to control levels has been associated with a regulatory mechanism to control the wound response duration (León et al., 2001; Thornburg and Li, 1991). Additional research would be needed to better understand these results for ZR and IAA in turfgrass species.

GA isoforms $\mathrm{GA}_{1}$ and $\mathrm{GA}_{20}$ were generally upregulated due to heat stress in most trimming treatments, especially for 'Penn-G2'. $\mathrm{GA}_{4}$ was not greatly affected by trimming frequency (either no trimming or trimming every $3 \mathrm{~d}$ ). $\mathrm{GA}_{4}$ may accumulate within minutes after trimming of creeping bentgrass leaves, as demonstrated by the increase in $\mathrm{GA}_{4} 15$ or 30 min after leaf trimming on several sampling days. GA has been associated with heat tolerance in some plant tissues and inhibition of GA by plant growth regulators has been shown to reduce heat tolerance (Alonso-Ramírez et al., 2009; Heckman et al., 2002). It is not clear whether GAs are or how GAs may be related to heat stress in the results of this study. Relative to the other hormones, less information is available regarding GA involvement in plant leaf wounding responses. GA has been found to negatively regulate some wound responses, such as the accumulation of mitogen-activated protein 

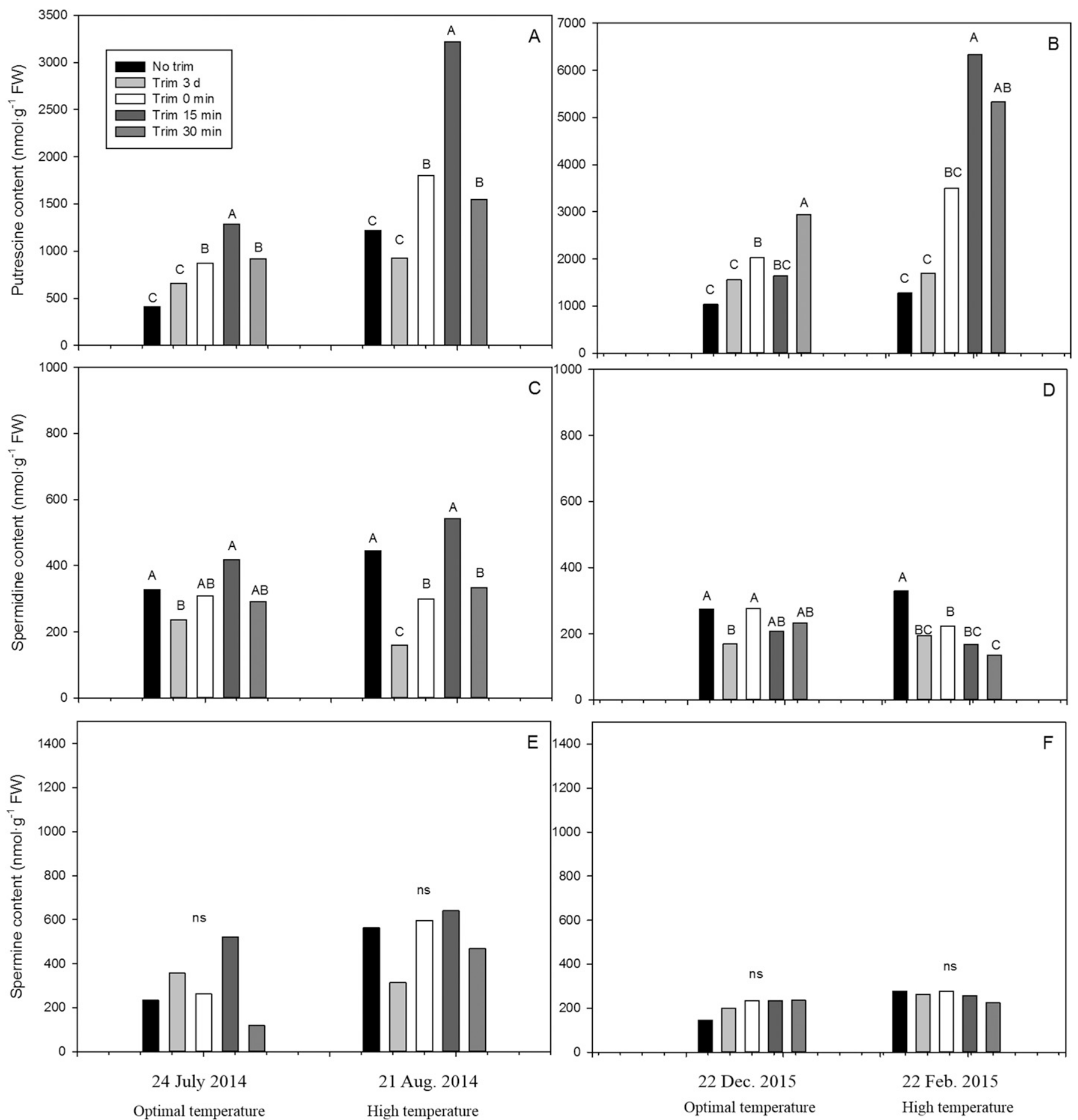

Fig. 5. Polyamine content of creeping bentgrass leaves exposed to trimming treatments combined with optimal or high temperature conditions including putrescine content in (A) Expt. 1 'Penncross' and (B) Expt. 2 'Penn-G2'; spermidine content in (C) Expt. 1 'Penncross' and (D) Expt. 2 'Penn-G2'; and spermine content in (E) Expt. 1 'Penncross' and (F) Expt. 2 'Penn-G2'. Different letters indicate significant differences among leaf trimming treatments within a given day. Asterisks indicate significant differences within a treatment comparing among temperature conditions. Letters and asterisks are based on separation of means using Fisher's protected least significant difference $(\mathrm{n}=6)$ at $P \leq 0.05$. Note scale differences on $y$ axis for all graphs used to visualize treatment differences.

kinase gene transcripts (Bogre et al., 1997). Thus, why different isoforms may have been regulated due to trimming or heat stress in creeping bentgrass leaves may deserve further study.

The changes in PA content after wounding at 0 to $30 \mathrm{~min}$ are relatively consistent with what is observed in other species. We found no consistent trend in Spd content between experiments in response to wounding and no significant changes due to any treatment in Spm content. A transient increase in Put was observed at $15 \mathrm{~min}$ after wounding. In arabidopsis (Arabidopsis thaliana) leaves, there was a sustained but transient increase in Put (elevated level from 2 to $10 \mathrm{~h}$ ) after wounding, no significant change in Spd, and a mild decrease in Spm (Perez-Amador et al., 2002). Relative 
to these species, it seems that creeping bentgrass could have quicker shifts in Put content that can be measured in minutes, not hours. This could be related to the evolutionary requirement of grasses to quickly heal wounded leaves during frequent herbivory, but this cannot be directly concluded from these results. PAs were elevated for $\approx 24 \mathrm{~h}$ in potato (Solanum tuberosum) tubers throughout the wound healing process, including during formation of the closing layer through to when cells became meristematic to form the wound cell layer (Lulai et al., 2015). Little information is available on wound healing specifically in turfgrass leaves. It is not clear from the results of this study whether Spd and Spm content may play a role in wounding responses in creeping bentgrass.

In regard to $\mathrm{PA}$ content comparing nontrimmed plants to plants trimmed every $3 \mathrm{~d}$, there were no significant changes in Put content. Spd content was significantly reduced in both experiments and within both temperature treatments and no significant changes were detected for Spm. Thus, more frequent trimming or mowing could moderate Spd accumulation in creeping bentgrass leaves. This could play a role in tolerance to abiotic or biotic stresses, since Spd is widely known to be associated with promoting stress tolerance (Hussain et al., 2011). Exogenous application of Spd was effective in promoting drought tolerance in creeping bentgrass (Shukla et al., 2015). More detailed evaluation of PA responses to mowing frequency would be needed to make any specific conclusions based on this finding.

In conclusion, imposition of different trimming practices including no trimming, trimming once per week, or trimming every $3 \mathrm{~d}$ resulted in significantly different accumulation of plant hormones profiles. As plants trimmed once per week were often comparable to plants trimmed every $3 \mathrm{~d}$ and both often differed from untrimmed plants, it seems that both infrequent and frequent modification of the leaf canopy had significant impact on hormone accumulation. Time course evaluation after leaf cutting to evaluate wounding responses at 0,15 , and $30 \mathrm{~min}$ postcutting demonstrated that a significant accumulation of JA and IAA occurred as well as differential regulation of GA isoforms and a transient accumulation of Put. Heat stress tended to increase levels of SA, ABA, IAA, GA, $\mathrm{GA}_{20}$, and Put in most treatments, whereas a decline in $\mathrm{ZR}$ was observed.

\section{Literature Cited}

Alonso-Ramírez, A., D. Rodríguez, D. Reyes, J.A. Jiménez, G. Nicolás, M. López-Climent, A. Gómez-Cadenas, and C. Nicolás. 2009. Evidence for a role of gibberellins in salicylic acid-modulated early plant responses to abiotic stress in arabidopsis seeds. Plant Physiol. 150:1335-1344.

Alvim, R., E.W. Hewett, and P.F. Saunders. 1976. Seasonal variation in the hormone content of willow: I. Changes in abscisic acid content and cytokinin activity in the xylem sap. Plant Physiol. 57:474-476.

Backman, P., G. Stahnke, and E. Miltner. 2002. Anthracnose update: Cultural practices affect spread of disease in Northwest. Turfgrass Trends. 11(10):T1-T4.

Beard, J.B. 2002. Turf management for golf courses. 2nd ed. Ann Arbor Press, Chelsea, MI.

Bogre, L., W. Ligterink, I. Meskiene, P.J. Barker, E. Heberle-Bors, N.S. Huskisson, and H. Hirt. 1997. Wounding induces the rapid and transient activation of a specific MAP kinase pathway. Plant Cell 9:75-83.

Crane, K.E. and C.W. Ross. 1986. Effects of wounding on cytokinin activity in cucumber cotyledons. Plant Physiol. 82:1151-1152.
Davies, P.J. 1995. Plant hormones. Springer, Dordrecht, The Netherlands.

Delvalle, T.C., P.J. Landschoot, and J.E. Kaminski. 2011. Effects of dew removal and mowing frequency on fungicide performance for dollar spot control. Plant Dis. 95:1427-1432.

Doares, S.H., J. Narvaez-Vasquez, A. Conconi, and C.A. Ryan. 1995. Salicylic acid inhibits synthesis of proteinase inhibitors in tomato leaves induced by systemin and jasmonic acid. Plant Physiol. 108:1741-1746.

Fu, Z.Q. and X. Dong. 2013. Systemic acquired resistance: Turning local infection into global defense. Annu. Rev. Plant Biol. 64:839863.

He, Y., Y. Liu, W. Cao, M. Huai, B. Xu, and B. Huang. 2005. Effects of salicylic acid on heat tolerance associated with antioxidant metabolism in kentucky bluegrass. Crop Sci. 45:988-995.

Heckman, N.L., G.L. Horst, R.E. Gaussoin, and B.T. Tavener. 2002. Trinexapac-ethyl influence on cell membrane thermostability of kentucky bluegrass leaf tissue. Sci. Hort. 92:183-186.

Hoagland, D.R. and D.I. Arnon. 1950. The water-culture method for growing plants without soil. Univ. California, College Agr., Agr. Expt. Sta. Circ. 347.

Hussain, S.S., M. Ali, M. Ahmad, and K.H. Siddique. 2011. Polyamines: Natural and engineered abiotic and biotic stress tolerance in plants. Biotechnol. Adv. 29:300-311.

Inguagiato, J.C., J.A. Murphy, and B.B. Clarke. 2009. Anthracnose disease and annual bluegrass putting green performance affected by mowing practices and lightweight rolling. Crop Sci. 49:14541462.

Kotak, S., J. Larkindale, U. Lee, P. von Koskull-Döring, E. Vierling, and K.D. Scharf. 2007. Complexity of the heat stress response in plants. Curr. Opin. Plant Biol. 10:310-316.

Krishnan, S.K. and E. Merewitz. 2015a. Drought stress and trinexapacethyl modify phytohormone content within kentucky bluegrass leaves. J. Plant Growth Regul. 34:1-12.

Krishnan, S.K. and E. Merewitz. 2015b. Phytohormone responses and cell viability during salinity stress in two creeping bentgrass cultivars differing in salt tolerance. J. Amer. Soc. Hort. Sci. 140:346-355.

Larkindale, J. and B. Huang. 2004. Thermotolerance and antioxidant systems in Agrostis stolonifera: Involvement of salicylic acid, abscisic acid, calcium, hydrogen peroxide, and ethylene. J. Plant Physiol. 161:405-413.

Lee, A., K. Cho, S. Jang, R. Rakwal, H. Iwahashi, G.K. Agrawal, J. Shim, and O. Han. 2004. Inverse correlation between jasmonic acid and salicylic acid during early wound response in rice. Biochem. Biophys. Res. Commun. 318:734-738.

León, J., E. Rojo, and J.J. Sánchez-Serrano. 2001. Wound signaling in plants. J. Expt. Bot. 52:1-9.

Liu, H., X. Li, J. Xiao, and S. Wang. 2012. A convenient method for simultaneous quantification of multiple phytohormones and metabolites: application in study of rice-bacterium interaction. Plant Methods 8:2.

Liu, R., Y. Jia, W. Cheng, J. Ling, L. Liu, K. Bi, and Q. Li. 2011. Determination of polyamines in human urine by precolumn derivatization with benzoyl chloride and high-performance liquid chromatography coupled with Q-time-of-flight mass spectrometry. Talanta 83:751-756.

Lulai, E.C., J.D. Neubauer, L.L. Olson, and J.C. Suttle. 2015. Wounding induces changes in tuber polyamine content, polyamine metabolic gene expression, and enzyme activity during closing layer formation and initiation of wound periderm formation. J. Plant Physiol. 176:89-95.

Nováková, M., V. Motyka, P.I. Dobrev, J. Malbeck, A. Gaudinová, and R. Vanková. 2005. Diurnal variation of cytokinin, auxin and abscisic acid levels in tobacco leaves. J. Expt. Bot. 56:2877-2883.

Oefner, P.J., S. Wongyai, and G. Bonn. 1992. High-performance liquid chromatographic determination of free polyamines in human seminal plasma. Clin. Chim. Acta 205:11-18. 
Papenfus, H.B., W.A. Stirk, J.F. Finnie, and J. Van Staden. 2012. Seasonal variation in the polyamines of Ecklonia maxima. Bot. Mar. 55:539-546.

Perez-Amador, M.A., J. Leon, P.J. Green, and J. Carbonell. 2002. Induction of the arginine decarboxylase $\mathrm{ADC} 2$ gene provides evidence for the involvement of polyamines in the wound response in arabidopsis. Plant Physiol. 130:1454-1463.

Petrásek, J. and J. Friml. 2009. Auxin transport routes in plant development. Development 136:2675-2688.

Putman, A.I. and J.E. Kaminski. 2011. Mowing frequency and plant growth regulator effects on dollar spot severity and on duration of dollar spot control by fungicides. Plant Dis. 95:1433-1442.

Rajendran, S.K., I.W. Lin, M.J. Chen, C.Y. Chen, and K.W. Yeh. 2014. Differential activation of sporamin expression in response to abiotic mechanical wounding and biotic herbivore attack in the sweet potato. BMC Plant Biol. 14:112-133.

Rossi, F.R., M. Marina, and F.L. Pieckenstain. 2015. Role of arginine decarboxylase (ADC) in Arabidopsis defense against the pathogenic bacterium Pseudomonas viridiflava. Plant Biol. 17:831-839.
Sanchez-Serrano, J.J. 2010. Plant responses to wounding, p. 1-5. In: A.M. Hetherington (ed.). Encyclopedia of life sciences. Wiley, Chichester, UK.

Shukla, V., Y. Ma, and E. Merewitz. 2015. Creeping bentgrass responses to drought stress and polyamine application. J. Amer. Soc. Hort. Sci. 140:94-101.

Thornburg, R.W. and X. Li. 1991. Wounding Nicotiana tabacum leaves causes a decline in endogenous indole-3-acetic acid. Plant Physiol. 96:802-805.

Ton, J., J.A. Van Pelt, L.C. Van Loon, and C.M. Pieterse. 2002. Differential effectiveness of salicylate-dependent and jasmonate/ ethylene-dependent induced resistance in arabidopsis. Mol. Plant Microbe Interact. 15:27-34.

Turgeon, A.J. 2011. Turfgrass management. 9th ed. Pearson Prentice Hall, Upper Saddle River, NJ.

Uddin, W. and M.D. Soika. 2003. Effects of turfgrass cultural management practices on severity of anthracnose basal rot in mixedannual bluegrass and creeping bentgrass greens. Phytopathology 93:S86.

Wang, K.L.C., H. Li, and J.R. Ecker. 2002. Ethylene biosynthesis and signaling networks. Plant Cell 14:S131-S151. 